\title{
Comparative Study of Three Pretreatment Methods on Sugarcane
}

\section{Bagasse}

$$
\begin{gathered}
\text { Lihong Cui }{ }^{1, \mathrm{a}} \text {, Jihua } \mathrm{Li}^{1, \mathrm{~b}^{*}} \text {, Xiaoyi Wei }{ }^{1, \mathrm{c}} \text {, Bing Tang } \\
\text { 2,d } \\
\qquad \mathrm{Wu}^{3, \mathrm{f}}
\end{gathered}
$$

1 Key Laboratory of Tropical Crop Products Processing of Ministry of Agriculture, Agriculture Products Processing Research Institute, Chinese Academy of Tropical Agricultural Sciences, Zhanjiang 524001, China

2 Spice and Beverage Research Institute, Chinese Academy of Tropical Agricultural Sciences, Wanning 571533, China

3 South Subtropical Crops Research Institute, Chinese Academy of Tropical Agricultural Sciences, Zhanjiang 524001, China

a'LH_cui@126.com, bfoodpaper@126.com, 'weixywei@163.com, dangb@catas.cn, echanggang86@126.com, 'whd1968@163.com

Keywords: Cellulose; Sugarcane bagasse; Pretreatment; Enzymatic hydrolysis; Acid Coupled Alkaline Pretreatment

Abstract. Pretreatment of lignocellulose is a key step in a commercial biorefinery. To increase the cellulose mass fractions and enhance the enzymatic hydrolysis, sugarcane bagasse was pretreated with methods of dilute acid, dilute alkaline and acid coupled alkaline. The efficiency of these three methods is compared. After pretreated with acid coupled alkaline, the cellulose mass fraction in sugarcane bagasse increased from $54.43 \% \%$ to $93.53 \%$. The initial hydrolysis rate for acid coupled alkaline treated sample was up to 15.13 times greater than those of untreated one. Thus, acid coupled alkaline pretreatment is a promising method for enhancing enzymes accessibility of lignocellulose.

\section{Introduction}

Cellulose is receiving increased attention due to its potential use in bioenergy production [1]. However, these polysaccharide molecules are not readily hydrolysed since they are contained in the chemically recalcitrant and structurally complex lignocellulosic matrix [2]. Pretreatment is a necessary step to disrupt the tight structure of lignocellulosic biomass, for enhancing enzymes accessibility to cellulose during hydrolysis. Comminution, steam explosion, ammonia fiber explosion, and acid or alkaline pretreatment processes have been extensively investigated [3]. Acid pretreatment generally use sulfuric, nitric, or hydrochloric acids to remove hemicellulose components, so that expose cellulose for enzymatic digestion [4]. Alkali pretreatment refers to the application of alkaline solutions to remove lignin and various uronic acid substitutions on hemicellulose that lower the accessibility of enzyme to the cellulose [5]. But traditional pretreatment processes are severe and not efficient enough.

The purpose of this study was to investigate the effect of different pretreatment methods using 
dilute acid, dilute alkaline or acid coupled alkaline on the components and the following enzymatic hydrolysis of sugarcane bagasse, so that a chemical pretreatment method of lignocellulose under mild conditions will be developed.

\section{Experimental Section}

Materials. Sugarcane bagasse was obtained from a local sugar factory (Zhanjiang, China). It was air-dried, milled, screened to obtain the particles of 40-80 mesh, and then stored until used. Cellulase from Aspergillus niger was purchased from Sukahan Bio-tech Co., Ltd.

Dilute Acid Pretreatment. The sugarcane bagasse powder was soaked in 3 wt $\%$ nitric acid with a solid to liquor ratio of $1: 15$, extract hemicellulose for $3 \mathrm{~h}$ at $100 \square$.

Dilute Alkaline Pretreatment. The sugarcane bagasse powder was soaked in $1.5 \mathrm{wt} \%$ sodium hydrate with a solid to liquor ratio of $1: 15$, and delignified for $1.5 \mathrm{~h}$ at $100 \square$.

Acid Coupled Alkaline Pretreatment. To obtain sugarcane bagasse cellulose, dilute alkaline pretreatment was conduct following the dilute acid pretreatment.

After pretreatment, the solid fraction (pretreated bagasse) was washed with deionized water until neutrality, and then freeze-dried. A portion of the pretreated bagasse was stored for subsequent chemical composition analysis, and the rest of the material was used for enzymatic hydrolysis.

Chemical Composition Analysis. The content of cellulose, hemicellulose and lignin in raw and pretreated sugarcane bagasse was determined according to Van Soest method [6].

Surface Morphology Analysis. The morphology analysis of raw and pretreated sugarcane bagasse was done by scanning electron microscopy (SEM). The SEM pictures were taken at different magnifications using environmental scanning electron microscope model QUANTA250 (FEI, US) with an accelerating voltage of $20 \mathrm{kV}$. The samples were coated with $20 \mathrm{~nm}$ of gold and kept in a desiccator until analysis.

Enzymatic Hydrolysis. The commercial cellulase activity was 180FPU per gram of original enzyme power, which determined using standard protocol from the National Renewable Energy Laboratory (NREL) (www.nrel.org). Batch enzymatic hydrolysis of pretreated and untreated sugarcane bagasse samples was carried out at $50{ }^{\circ} \mathrm{C}$ in a reciprocating shaker bath. All samples were diluted to $16 \mathrm{~g} / \mathrm{L}$ in a $0.05 \mathrm{M}$ citrate buffer with $\mathrm{pH} 4.8$ for enzymatic hydrolysis. The total batch volume was $2 \mathrm{~mL}$ with cellulase concentration of 14 FPU per gram substrate. The reaction was monitored by withdrawing samples from the supernatant periodically, and measuring the released reducing sugars by the DNS assay using D-glucose as a standard [7]. The initial rates of total soluble reducing sugar formation are calculated based on the analysis of supernatant sampled during the first 20 min of hydrolysis [8].

\section{Results and Discussion}

Chemical Composition Analysis. The effect of three different pretreatment on the chemical composition of sugarcane bagasse was shown in Fig. 1. The high cellulose content $(54.43 \%)$ in the raw sugarcane bagasse indicates the high potential of the material for producing biofuel because cellulose is source of fermentable sugar. But this material needs pretreatment to remove hemicelluloses and lignin and increase its digestibility for subsequent enzymatic hydrolysis.

Results show that when compared with the untreated sample, both dilute acid and dilute alkaline pretreatment made cellulose content in sugarcane bagasse increase, ranging from $64.89 \%$ to 78.27\%. After dilute acid pretreatment, hemicellulose content decreased sharply. In comparison, 
dilute alkaline pretreatment remove more lignin than dilute acid pretreatment. These results indicate that dilute acid pretreatment results in removing hemicellulose effectively but dilute alkaline pretreatment results in significant level of delignification. For further increase cellulose component, acid coupled alkaline pretreatment was employed. It produced material with the lowest content of residual hemicellulose and lignin $(0.69 \%$ and $0.82 \%$, respectively) and the highest content of residual cellulose $(93.53 \%)$. Since hemicellulose and lignin are a physical barrier that surrounds cellulose fibers and protect them from enzymatic attack [9], their removal indicates the potential of the used method for activation of bagasse for enzymatic hydrolysis.

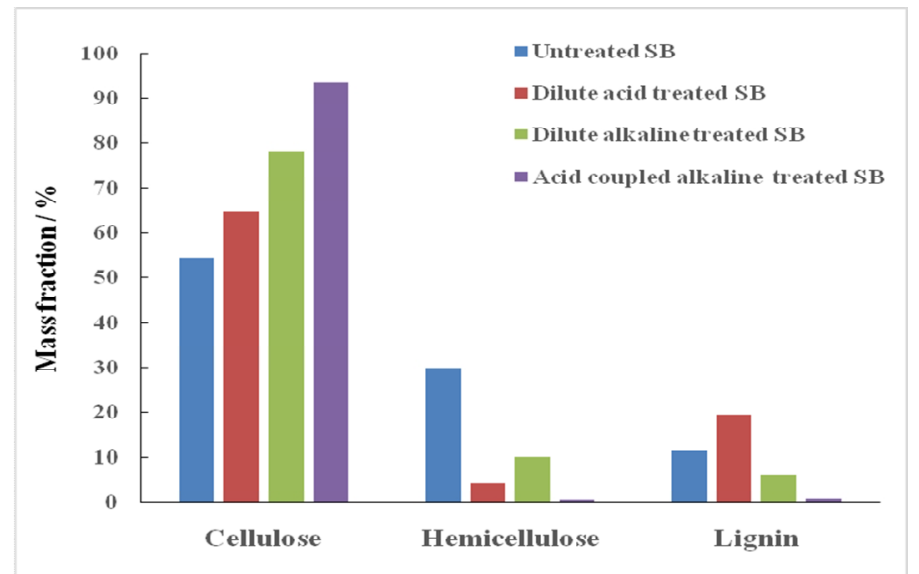

Fig.1 Chemical composition analysis of untreated and pretreated sugarcane bagasse (SB)

Surface Morphology Observation. SEM was a useful tool to investigate the surface structure of lignocellulosic biomass. Fig. 1 shows SEM images at 1500× magnifications of the raw and treated sugarcane bagasse samples. The untreated sugarcane bagasse has a tight structure with some pores on the surface. Compare to the untreated samples, the surface morphologies are only changed slightly by dilute acid and dilute alkaline pretreatment. The major structures are preserved, but there are cracks observed on the surface. However, acid coupled alkaline pretreatment significantly alters the surface structure. It can be attributed to the almost completely removal of hemicellulose and lignin in sugarcane bagasse after being treated by acid coupled alkaline, thus interrupted the cross linking between cellulose, hemicellulose and lignin. This morphological structure could suggest a large accessible surface to the cellulase, which was in agreement with the reducing sugar yield obtained. These results are consistent with the observations that faster hydrolysis rates are obtained for acid coupled alkaline pretreated sample than for others, which will be discussed as follow.
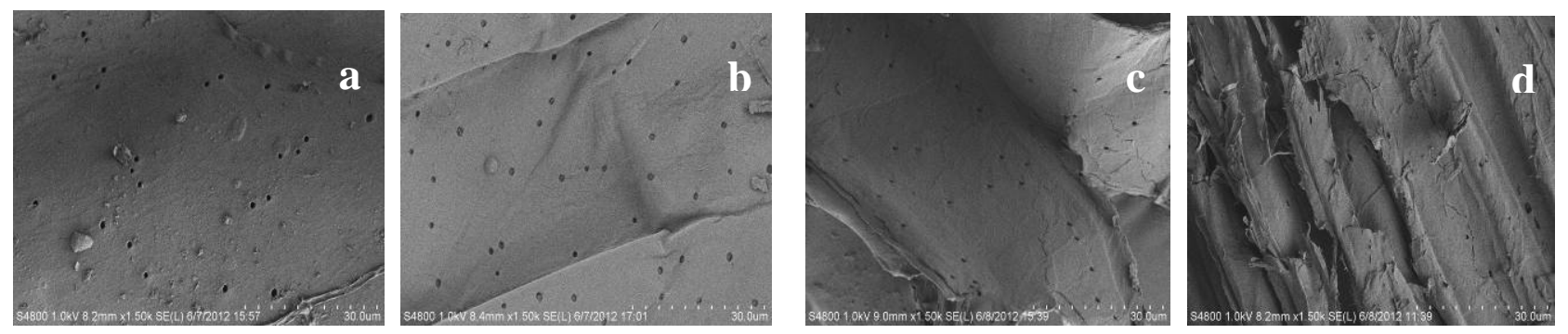

Fig.2 SEM images of (a) untreated, (b) pretreated with dilute acid, (c) dilute alkaline and (d) acid coupled alkaline sugarcane bagasse samples

Enzymatic Hydrolysis. Enzymatic hydrolysis of untreated and pretreated sugarcane bagasse was carried out to compare their initial rate of soluble reducing sugar formation. The results are 
shown in Table 1. Initial rate of enzymatic hydrolysis of untreated sugarcane bagasse was only $0.0099 \mathrm{mg} / \mathrm{mL} / \mathrm{min}$. After treated by dilute acid and dilute alkaline, initial rate of enzymatic hydrolysis increased slightly as seen in Table 1. Acid coupled alkaline pretreated sugarcane bagasse exhibited significantly higher initial rate which were up to 15.13 times greater than untreated sample. This implies that it is necessary to remove hemicellulose and lignin to achieve significant enhancement in hydrolysis rates.

Table 1 Comparison of three pretreatment methods on initial rate of enzymatic hydrolysis

\begin{tabular}{lcc}
\hline & $\begin{array}{c}\text { Initial rate } \\
{[\mathrm{mg} / \mathrm{mL} / \mathrm{min}]}\end{array}$ & Rate enhancement $^{\mathrm{a}}$ \\
\hline Untreated SB & 0.0099 & - \\
Dilute acid treated SB & 0.0422 & 4.26 \\
Dilute alkaline treated SB & 0.0190 & 1.92 \\
Acid coupled alkaline treated SB & 0.1498 & 15.13 \\
\hline
\end{tabular}

${ }^{a}$ Rate enhancement is defined as the ratio of initial rate of reducing sugars released for pretreated sample divided by that of untreated sample.

\section{Conclusions}

In this study, a comparison of dilute acid, dilute alkaline and acid coupled alkaline pretreatment on chemical component and enzymatic hydrolysis of sugarcane bagasse was conducted. Compared with the other two pretreatments, acid coupled alkaline pretreatment resulted in significantly higher cellulose mass fractions and produced significant enhancement of enzyme hydrolysis kinetics. Since hemicellulose and lignin was almost clearly removed, the surface morphologies of sugarcane bagasse treated by acid coupled alkaline showed important change which could suggest a large accessible surface to the cellulase. The results indicate that the acid coupled alkaline pretreatment is a promising method for lignocellulosic biorefinery.

\section{Acknowledgments}

The authors are extremely grateful for the financial support from Hainan Natural Science Foundation (No. 313051), National Nonprofit Institute Research Grant of CATAS (No. 1630062013012 \& No.1630122017018) and Zhanjiang agricultural research project (No. 2016A03019).

\section{References}

[1] Park S, Baker J O, Himmel M E, et al. Cellulose crystallinity index: measurement techniques and their impact on interpreting cellulase performance [J]. Biotechnology for Biofuels, 2010, 3(1): 10 .

[2] Karatzos S K, Edye L A, Doherty W O S. Sugarcane bagasse pretreatment using three imidazolium-based ionic liquids; mass balances and enzyme kinetics [J]. Biotechnology for Biofuels, 2012, 5(1): 62.

[3] Sun Y, Cheng J J. Dilute acid pretreatment of rye straw and bermudagrass for ethanol production [J]. Bioresource Technology, 2005, 96(14): 1599-1606. 
[4] Silverstein R A, Chen Y, Sharma-Shivappa R R, et al. A comparison of chemical pretreatment methods for improving saccharification of cotton stalks [J]. Bioresource Technology, 2007, 98(16): 3000-3011.

[5] Chang V S, Holtzapple M T. Fundamental factors affecting biomass enzymatic reactivity [J]. Applied Biochemistry and Biotechnology, 2000, 84(1): 5-37.

[6] Van Soest PJ, Robertson JB, Lewis BA. Methods for dietary fiber, neutral detergent fiber, and nonstarch polysaccharides in relation to animal nutrition [J]. Journal of Dairy Science, 1991, 74(10): 3583-3597.

[7] Miller G L. Use of dinitrosalicylic acid reagent for determination of reducing sugar[J]. Anal Chem, 1959, 31: 426-42.

[8] Dadi A P, Schall C A, Varanasi S. Mitigation of cellulose recalcitrance to enzymatic hydrolysis by ionic liquid pretreatment [J]. Applied Biochemistry and Biotechnology, 2007, 137(1): 407-421.

[9] Taherzadeh M J, Karimi K. Pretreatment of lignocellulosic wastes to improve ethanol and biogas production: a review[J]. Int J Mol Sci, 2008, 9: 1621-51. 